\title{
On the Reversal of Myocardial Stunning: A Role for $\mathrm{Ca}^{2+}$-Sensitizers
}

\author{
DONGSHENG FAN, LOE KIE SOEI, \\ LOES M. A. SASSEN, ROB KRAMS, \\ EDWIN HENDRIK, AND \\ PIETER D. VERDOUW ${ }^{a}$ \\ Experimental Cardiology, Thoraxcenter \\ Cardiovascular Researsh Institute (COEUR) \\ Erasmus University Rotterdam \\ P.O. Box 1738 \\ 3000 DR Rotterdam \\ The Netherlands
}

\section{INTRODUCTION}

The mechanism underlying myocardial stunning is still unknown, but current views hold that generation of free radicals and disturbances in the calcium homeostasis, mechanisms which are not mutually exclusive, are the two most likely causes of prolonged postischemic dysfunction.1,2 Several groups of investigators have indeed shown that the capacity of cardiac sarcoplasmic reticulum (SR) to sequester $\mathrm{Ca}^{2+}$ decreases during ischemia. ${ }^{3,4}$ In a recent study we have shown that the phosphorylation rate of phospholamban was unchanged, and that $\mathrm{Ca}^{2+}$ uptake by the sarcoplasmic reticulum was even slightly increased in myocardium of intact open-chest pigs, while function was still reversibly depressed. ${ }^{5}$ These data and earlier work by Marban and coworkers $^{6}$ in isolated stunned ferret hearts strongly suggest that a decreased response of the myofilaments to $\mathrm{Ca}^{2+}$ rather than a change in the active $\mathrm{Ca}^{2+}$ transport of the $\mathrm{SR}$ is involved in the contractile dysfunction of stunned myocardium. In vivo evidence of this hypothesis has been very difficult to obtain because available agents that increase the responsiveness of the myofilaments to $\mathrm{Ca}^{2+}$ usually also increase contractility via inhibition of phosphodiesterase. We now report on the effects of the thiadiazinone derivative EMD 60263, which in in vitw experiments has been shown to be a potent $\mathrm{Ca}^{2+}$ sensitizer devoid of any phosphodiesterase inhibiting properties (Ravens et al., unpublished data). The compound also affects the delayed rectifier current $I_{K r}$ in a way characteristic for a class III antiarrhythmic action. The latter property may lead to a reduction in heart rate.

a To whom correspondence should be addressed. 


\section{MATERIALS AND METHODS}

Pigs $\left(28-30 \mathrm{~kg}\right.$ ) were anesthetized and instrumented as described earlier. ${ }^{7}$ After a stabilization period the left anterior descending coronary artery (LADCA) was occluded for $10 \mathrm{~min}$ and reperfused for $30 \mathrm{~min}$. This sequence of occlusion and reperfusion was then repeated. At the end of the second 30-min reperfusion period, two consecutive infusions of either saline $(3 \mathrm{ml}$ and $6 \mathrm{ml} ; n=6)$ or EMD $60263(1.5 \mathrm{mg} / \mathrm{kg}$ and $3.0 \mathrm{mg} / \mathrm{kg}, n=7$; courtesy of Dr. I. Lues and Prof. Dr. P. Schelling, E. Merck, Darmstadt, FRG) were administered at 15-min intervals and the effects on systemic hemodynamics, regional myocardial segment length shortening (SLS) of the myocardium perfused by the LADCA and the left circumflex coronary artery (LCXCA), and regional myocardial blood flow were measured. The area of the pressure-segment length loop was calculated as an index of external work (EW), ${ }^{8}$ while the mechanical efficiency was determined as the ratio of EW and $\mathrm{MVO}_{2}$, in which the $\mathrm{MVO}_{2}$ is the regional myocardial oxygen consumption (i.e., the product of regional transmural myocardial blood flow and the difference in the arterial and local coronary venous oxygen content).

TABLE 1. The Effects of EMD 60263 on Systemic Hemodynamics in Anesthetized Pigs with Stunned Myocardium

\begin{tabular}{clcccc}
\hline & & & $3 \mathrm{ml}$ & $6 \mathrm{ml}$ \\
& EMD 60263 & Baseline & Stunning & $1.5 \mathrm{mg} / \mathrm{kg}$ & $3.0 \mathrm{mg} / \mathrm{kg}$ \\
\hline Heart rate & Saline & $107 \pm 3$ & $103 \pm 7$ & $103 \pm 8$ & $101 \pm 8$ \\
(beats/min) & EMD 60263 & $107 \pm 5$ & $97 \pm 3^{*}$ & $69 \pm 2^{\dagger \#}$ & $50 \pm 3^{\dagger \#}$ \\
Systolic arterial & Saline & $109 \pm 2$ & $101 \pm 3^{*}$ & $101 \pm 2$ & $107 \pm 3^{\dagger}$ \\
pressure (mm Hg) & EMD 60263 & $114 \pm 2$ & $108 \pm 3$ & $106 \pm 3$ & $111 \pm 4$ \\
Diastolic arterial & Saline & $77 \pm 2$ & $73 \pm 3^{*}$ & $72 \pm 2$ & $75 \pm 2$ \\
pressure (mm Hg) & EMD 60263 & $83 \pm 3$ & $79 \pm 4$ & $67 \pm 4^{\dagger \#}$ & $62 \pm 3^{\dagger \#}$ \\
Cardiac output & Saline & $2.9 \pm 0.3$ & $2.4 \pm 0.1^{*}$ & $2.4 \pm 0.2$ & $2.4 \pm 0.0^{\dagger}$ \\
$\begin{array}{c}\text { (L/min) } \\
\text { Stroke volume }\end{array}$ & EMD 60263 & $2.9 \pm 0.1$ & $2.5 \pm 0.1^{*}$ & $2.3 \pm 0.1$ & $2.2 \pm 0.1^{\dagger}$ \\
(ml) & Saline & $27 \pm 2$ & $23 \pm 1$ & $23 \pm 1$ & $24 \pm 1$ \\
Systemic vascular & EMD 60263 & $27 \pm 2$ & $26 \pm 1$ & $33 \pm 2^{\dagger \#}$ & $45 \pm 3^{\dagger \#}$ \\
resistance & Saline & $32 \pm 3$ & $35 \pm 2^{*}$ & $35 \pm 3$ & $36 \pm 3$ \\
$\begin{array}{c}\text { (mm Hg-min/L) } \\
\text { Left ventricular }\end{array}$ & EMD 60263 & $33 \pm 3$ & $36 \pm 3$ & $36 \pm 3$ & $36 \pm 3$ \\
dP/dt $m a x$ & Saline & $2070 \pm 220$ & $1580 \pm 180^{*}$ & $1600 \pm 170$ & $1640 \pm 160$ \\
(mm Hg/sec) & EMD 60263 & $2130 \pm 150$ & $1580 \pm 110^{*}$ & $1520 \pm 110$ & $1630 \pm 100$ \\
Left ventricular end & Saline & $8 \pm 1$ & $9 \pm 1$ & $8 \pm 1$ & $10 \pm 2$ \\
diastolic pressure & EMD 60263 & $10 \pm 1$ & $10 \pm 1$ & $11 \pm 1$ & $14 \pm 2^{\dagger}$ \\
(mm Hg) & & & & & \\
\hline
\end{tabular}

$n=6$ for the saline-treated animals; $n=7$ for the EMD 60263 -treated animals; ${ }^{*} p<0.05$ stunning versus baseline; ${ }^{\dagger} p<0.05$ versus stunning; \# Changes versus stunning are significantly different from changes versus stunning in saline-treated animals; Data are mean \pm SEM. 

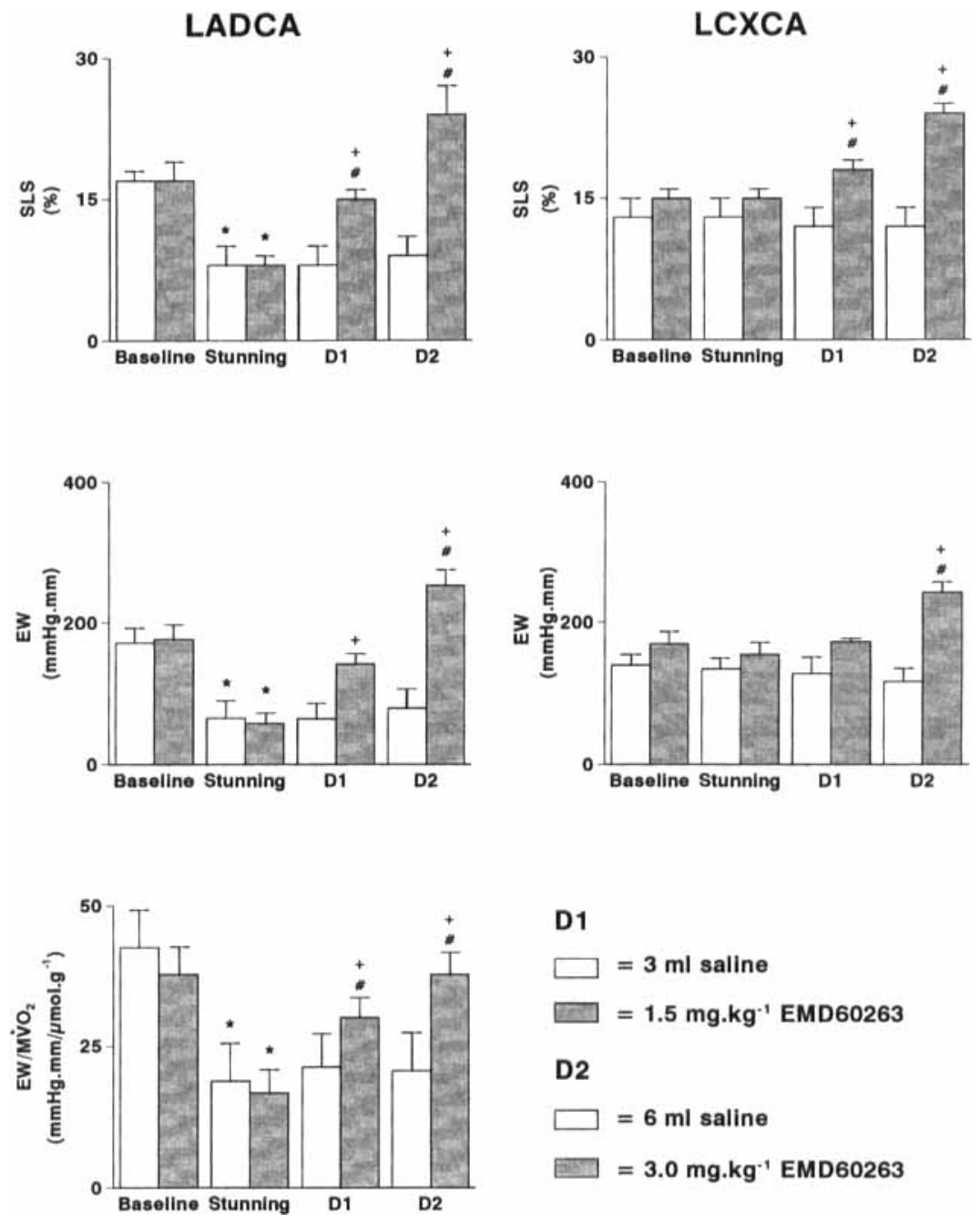

FIGURE 1. The effects of EMD 60263 on segment length shortening (SLS) and external work (EW) of the myocardium perfused by the left anterior descending coronary artery (LADCA) and the myocardium perfused by the left circumflex coronary artery (LCXCA) and the mechanical efficiency $\left(\mathrm{EW} / \mathrm{MVO}_{2}\right)$ of the LADCA-perfused myocardium. $n=6$ for the saline-treated animals; $n=7$ for the EMD 60263 -treated animals; ${ }^{*} p<0.05$ stunning versus baseline; ${ }^{+} p<0.05$ versus stunning; " changes versus stunning are significantly different from changes versus stunning in saline-treated animals. Data are mean \pm SEM. 


\section{RESULTS AND DISCUSSION}

Induction of myocardial stunning was accompanied by the well-described changes in systemic hemodynamic variables (TABLE 1 ). In the saline-treated animals there were no further changes in any of these variables during the following $30 \mathrm{~min}$. Administration of EMD 60263 caused a number of changes of which the decreases in heart rate and diastolic arterial pressure and the increase in stroke volume were the most pronounced.

An additional consequence of the occlusion-reperfusion protocol was that in the stunned myocardium systolic SLS, EW, and the $\mathrm{EW} / \mathrm{MVO}_{2}$ were reduced by approximately $50 \%$ (FrG. 1). No changes occurred in the area perfused by the LCXCA. All regional function variables remained stable in the saline-treated animals during the following $30 \mathrm{~min}$ (FIG. 1). In the stunned myocardium, administration of EMD 60263 caused dose-dependent increases in all variables, with the values for SLS and EW exceeding the data obtained at baseline. In the control segment some variables also increased, but, most importantly, all differences in the variables between the stunned and the control regions disappeared.

In order to exclude that changes in regional function were secondary to changes in heart rate or adrenergic stimulation, we studied in separate experiments, using the same model, the effects of the specific negative chronotropic drug zatebradine (UL-FS $49)^{9}$ and the effects of EMD 60263 after blockade of the $\alpha$ - and $\beta$-adrenoceptors and observed that bradycardia did not improve function of the stunned myocardium and that adrenergic stimulation did not contribute to the effect of EMD 60263.

We conclude that these experiments, although not providing definite proof, lend further support to the hypothesis that a decrease in the sensitivity of the myofilaments to $\mathrm{Ca}^{2+}$ plays a role in the mechanism leading to myocardial stunning.

\section{REPERENCES}

1. Hearse, D. J. 1991. Cardiovasc. Drugs Ther. 5: 853-876.

2. BOLLI, R 1990. Circulation 82: 723-738.

3. Krause, S. M., W. E. Jacobus \& L. C. Becker. 1989. Circ. Res. 65: 526-530.

4. Schoutsen, B., J. J. Blom, P. D. Verdouw \& J. M. J. Lamers. 1989. J. Mol. Cell Cardiol. 21: $719-727$.

5. Lamers, J. M. J., D. J. Duncker, K. Bezstarosti, E. O. McFalls, L. M. A. Sassen \& P. D. Verdouw. 1993. Cardiovasc. Res. 27: 520-524.

6. Marban, E., Y. Koretsune, M. Corretti, V. P. Chacko \& H. Kusuoka. 1989. Circulation 80: IV17-IV22.

7. Duncker, D. J., E. O. McFalls, R. Krams \& P. D. Verdouw. 1992. Am J. Physiol. 262 (Heart Circ. Physiol. 31): H1744-H1751.

8. Krams, R, D. J. Duncker, E. O. McFalls, A. Hogendoorn \& P. D. Verdouw. 1993. Cardiovasc. Res. 27: 740-747.

9. Van Woerkens, L. J., W. J. Van Der Giessen \& P. D. Verdouw. 1992. Cardiovasc. Drugs Ther. 6: 59-65. 